\title{
A semiempirical model for two-level system noise in superconducting microresonators
}

\author{
Jiansong Gao, ${ }^{1, a)}$ Miguel Daal, ${ }^{2}$ John M. Martinis, ${ }^{3}$ Anastasios Vayonakis, ${ }_{4}^{1}$ \\ Jonas Zmuidzinas, ${ }^{1}$ Bernard Sadoulet, ${ }^{2}$ Benjamin A. Mazin, ${ }^{4}$ Peter K. Day, ${ }^{4}$ and \\ Henry G. Leduc ${ }^{4}$ \\ ${ }^{1}$ Division of Physics, Mathematics, and Astronomy, California Institute of Technology, Pasadena, California \\ 91125, USA \\ ${ }^{2}$ Physics Department, University of California at Berkeley, Berkeley, California 94720, USA \\ ${ }^{3}$ Department of Physics, University of California, Santa Barbara, California 93106, USA \\ ${ }^{4}$ Jet Propulsion Laboratory, California Institute of Technology, Pasadena, California 91109, USA
}

(Received 2 April 2008; accepted 9 May 2008; published online 29 May 2008)

\begin{abstract}
We present measurements of the low-temperature excess frequency noise of four niobium superconducting coplanar waveguide microresonators, with center strip widths $s_{r}$ ranging from 3 to $20 \mu \mathrm{m}$. For a fixed internal power, we find that the frequency noise decreases rapidly with increasing center strip width, scaling as $1 / s_{r}^{1.6}$. We show that this geometrical scaling is readily explained by a simple semiempirical model which assumes a surface distribution of independent two-level system fluctuators. These results allow the resonator geometry to be optimized for minimum noise. (C) 2008 American Institute of Physics. [DOI: 10.1063/1.2937855]
\end{abstract}

Thin-film superconducting microresonators are of great interest for a number of applications (see Refs. 1-4 and references therein). Excess frequency noise is universally observed in these resonators ${ }^{2,5,6}$ and is very likely caused by two-level systems (TLSs) in dielectric materials. ${ }^{3,7}$ Indeed, the TLS hypothesis is supported by the observed dependence of the noise on resonator internal power ${ }^{7,8}$ and temperature. ${ }^{3}$ In a recent paper ${ }^{4}$ (paper A hereafter), we presented measurements of the TLS-induced low-temperature frequency shifts of five niobium $\left(T_{c}=9.2 \mathrm{~K}\right)$ coplanar waveguide (CPW) resonators with varying center strip widths $s_{r}$. From the observed geometrical scaling of the frequency shifts $\left(\sim 1 / s_{r}\right)$, we showed that the TLS must be located in a thin (few nanometer) layer on the surface of the CPW. In this letter, we propose a semiempirical TLS noise model that assumes this surface distribution, and we show that the model explains our measurements of the geometrical scaling of the noise.

The device used for the experiment in this paper is exactly the same device used in paper A. In brief, the chip contains five $\mathrm{CPW}$ quarter-wavelength resonators $\left(Z_{0}\right.$ $\approx 50 \Omega, f_{r} \approx 6 \mathrm{GHz}$ ) made by patterning a $120 \mathrm{~nm}$ thick $\mathrm{Nb}$ film deposited on a $c$-plane crystalline sapphire substrate. Each resonator is capacitively coupled to a common feedline, using a CPW coupler (coupling quality factor $Q_{c} \sim 50000$ ) of length $l_{c} \cong 200 \mu \mathrm{m}$ and with a common center-strip width of $s_{c}=3 \mu \mathrm{m}$. The coupler is then widened into the resonator body, with a center-strip width of $s_{r}=3,5,10,20$, or $50 \mu \mathrm{m}$, and a length of $l_{r} \sim 5 \mathrm{~mm}$. The noise was measured using a standard IQ homodyne technique; ${ }^{2,3}$ both the measurement setup and the analysis of the noise data are identical to our previous work. ${ }^{7}$

The device is cooled in a dilution refrigerator to a base temperature of $55 \mathrm{mK}$. The fractional frequency noise spec$\operatorname{tra} S_{\delta f}(\nu) / f_{r}^{2}$ of the five resonators were measured for microwave readout power $P_{\mu w}$ in the range -61 to $-73 \mathrm{dBm}$; the $-65 \mathrm{dBm}$ spectra are shown in Fig. 1(a). We clearly see that

${ }^{a)}$ Electronic mail: jiansong@caltech.edu. the noise has a common spectral shape but decreases as the center strip becomes wider. Unfortunately, the data for the lowest-noise $(50 \mu \mathrm{m})$ resonator are influenced by the noise floor of our cryogenic microwave amplifier, so we exclude this resonator from further discussion. The noise levels at $\nu=2 \mathrm{kHz}$ were retrieved from the noise spectra and are plotted as a function of resonator internal power $P_{\text {int }}$ $=2 Q_{r}^{2} P_{\mu w} / \pi Q_{c}$ in Fig. 1(b). All resonators display a power dependence close to $S_{\delta f} / f_{r}^{2} \propto P_{\text {int }}^{-1 / 2}$ as we have previously observed. ${ }^{3,7,8}$ In order to study the geometrical scaling of the noise in more detail, we first fit the noise versus power data for each resonator to a simple power law, and retrieve the values of the noise $S_{\delta f}(2 \mathrm{kHz}) / f_{r}^{2}$ at $P_{\text {int }}=-25 \mathrm{dBm}$ for each geometry. These results (Fig. 2) again show that the noise decreases with increasing $s_{r}$, although not (yet) as a simple power law.

To make further progress, we introduce a semiempirical model for the TLS noise. We assume that the TLS have a uniform spatial distribution within a volume of TLS-hosting

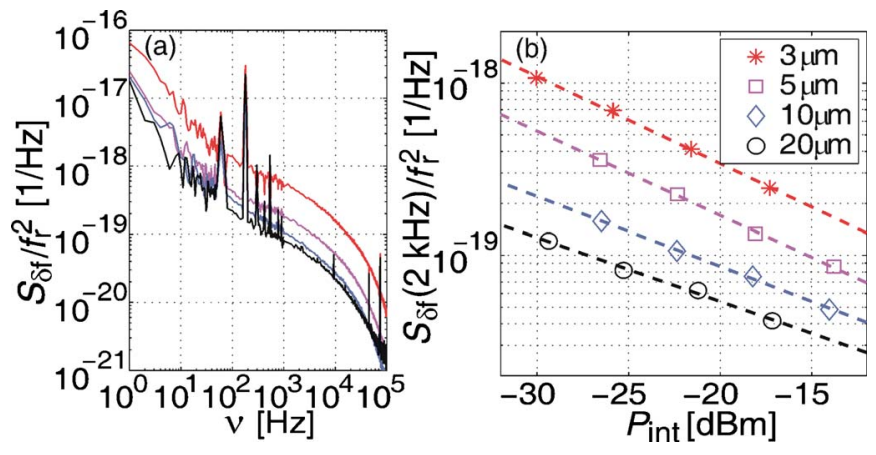

FIG. 1. (Color online) Fractional frequency noise spectra of the four CPW resonators measured at $T=55 \mathrm{mK}$. (a) Noise spectra at $P_{\mu w}=-65 \mathrm{dBm}$. From top to bottom, the four curves correspond to CPW center strip widths of $s_{r}=3,5,10$, and $20 \mu \mathrm{m}$. The various spikes seen in the spectra are due to pickup of stray signals by the electronics and cabling. (b) Fractional frequency noise at $\nu=2 \mathrm{kHz}$ as a function of $P_{\text {int }}$. The markers represent different resonator geometries, as indicated by the values of $s_{r}$ in the legend. The dashed lines indicate power law fits to the data of each geometry. 


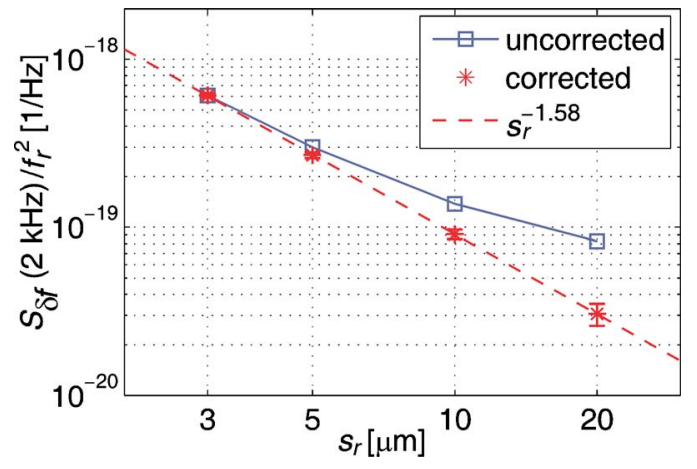

FIG. 2. (Color online) The measured fractional frequency noise $S_{\delta f}(2 \mathrm{kHz}) / f_{r}^{2}$ at $P_{\mathrm{int}}=-25 \mathrm{dBm}$ is plotted as a function of the center strip width $s_{r}$. Values directly retrieved from power-law fits to the data in Fig. 1 are indicated by the open squares. Values corrected for the coupler's contribution are indicated by the stars. The corrected values of $S_{\delta f}(2 \mathrm{kHz}) / f_{r}^{2}$ scale as $s_{r}^{-1.58}$, as indicated by the dashed line.

material $V_{h}$ that occupies some portion of the total resonator volume $V$. Consider a TLS labeled $\alpha$, located at a random position $\vec{r}_{\alpha} \in V_{h}$ and with an energy level separation $E_{\alpha}$ $=\left(\Delta_{\alpha}^{2}+\Delta_{0, \alpha}^{2}\right)^{1 / 2}$. Here $\Delta_{\alpha}$ and $\Delta_{0, \alpha}$ are the TLS asymmetry energy and tunnel splitting, which are random and have a joint distribution function $f\left(\Delta, \Delta_{0}\right)=P / \Delta_{0}$, where $P$ is the two-level density of states introduced by Phillips. ${ }^{9}$ The TLS transition dipole moment is given by $\vec{d}_{\alpha}=\hat{n}_{\alpha} d_{0} \Delta_{0, \alpha} / E_{\alpha}$, where $d_{0}$ is the maximum dipole moment for a TLS with energy $E_{\alpha}$ and the dipole orientation unit vector $\hat{n}_{\alpha}$ is assumed to be random and isotropically distributed. In the weak-field, linear response limit, the TLS contribution to the dielectric tensor of the hosting medium is

$$
\Delta \epsilon_{k l}(\omega, \vec{r})=-\sum_{\alpha} d_{\alpha, k} d_{\alpha, l} \delta\left(\vec{r}-\vec{r}_{\alpha}\right) \chi_{\alpha}(\omega) \sigma_{z, \alpha}
$$

where $k, l$ represent Cartesian components, $\chi_{\alpha}(\omega)=1 /\left(E_{\alpha}\right.$ $\left.-\hbar \omega+j \Gamma_{\alpha}\right)+1 /\left(E_{\alpha}+\hbar \omega-j \Gamma_{\alpha}\right)$ is a damped single-pole response function for $e^{+j \omega t}$ harmonic time dependence, and $\sigma_{z, \alpha}$ is the usual diagonal Pauli operator that takes values of -1 for the lower state of the TLS and +1 for the upper state. Averaging over the TLS position, asymmetry, tunnel splitting, and dipole orientation, and assuming a thermal distribution for the level population, the TLS contribution to the (isotropic) dielectric function is given by

$$
\begin{aligned}
\langle\Delta \epsilon(\omega)\rangle & =\int_{0}^{E_{\max }} \frac{P d_{0}^{2}}{3} \tanh \left(\frac{E}{2 k_{B} T}\right) \chi(\omega) d E \\
& =-\frac{2 P d_{0}^{2}}{3 \epsilon}\left[\Psi\left(\frac{1}{2}-\frac{\hbar \omega-j \Gamma}{2 j \pi k_{B} T}\right)-\log \frac{E_{\max }}{2 \pi k_{B} T}\right],
\end{aligned}
$$

where $\chi(\omega)=1 /(E-\hbar \omega+j \Gamma)+1 /(E+\hbar \omega-j \Gamma), E_{\max }$ is the maximum energy level separation, and $\Psi$ is the complex digamma function. The real $\left(\Delta \epsilon_{1}\right)$ and imaginary $\left(\Delta \epsilon_{2}\right)$ parts of Eq. (2) yield the well-known results for the TLS contribution to the dielectric constant ${ }^{10}$ and loss tangent. ${ }^{9,11}$ The former allows the temperature-dependent fractional frequency shift of a resonator to be computed using $\quad$ shift data. In the limit $l_{c} \ll l_{r}$, the correction is given by $S_{\delta f}^{*}$
Downloaded 02 Jun 2008 to 131.215 .225 .137 . Redistribution subject to AIP license or copyright; see http://apl.aip.org/apl/copyright.jsp

$$
\frac{\left\langle\Delta f_{r}\right\rangle}{f_{r}}=-\frac{\int_{V_{h}}\left\langle\Delta \epsilon_{1}\right\rangle|\vec{E}|^{2} d \vec{r}}{2 \int_{V} \epsilon|\vec{E}|^{2} d \vec{r}} .
$$

This result provides an excellent description of the experimental data, ${ }^{3,4}$ at $T \ll T_{c}$.

Now, if the dielectric constant fluctuates on time scales $\tau_{\epsilon} \gg 1 / \omega$, we would expect to see resonator frequency fluctuations given by

$$
\frac{\delta f_{r}(t)}{f_{r}}=-\frac{\int_{V_{h}} \delta \epsilon_{1}(\vec{r}, t)|\vec{E}|^{2} d \vec{r}}{2 \int_{V} \epsilon|\vec{E}|^{2} d \vec{r}} .
$$

From Eq. (1), we see that $\Delta \epsilon_{1}$ could fluctuate with time if the TLS switch states randomly $\left(\sigma_{z, \alpha}\right.$ changes sign), for instance due to phonon emission or absorption, or if the energy level separation $E_{\alpha}$ is perturbed randomly, for instance due to a collection of nearby TLS that randomly switch states and produce a randomly varying strain field that couples to TLS $\alpha$. Whatever the mechanism, for independently fluctuating TLS, from Eq. (1), we would expect the Fourier spectra of the $\delta \epsilon_{1}$ fluctuations to obey $\left\langle\delta \epsilon_{1}^{*}\left(\vec{r}_{1}, \nu_{1}\right) \delta \epsilon_{1}\left(\vec{r}_{2}, \nu_{2}\right)\right\rangle$ $=S_{\epsilon}\left(\vec{r}_{1}, \nu_{1}, T\right) \delta\left(\vec{r}_{1}-\vec{r}_{2}\right) \delta\left(\nu_{1}-\nu_{2}\right)$. Therefore, the resonator frequency power spectrum should be given by

$$
\frac{S_{\delta f}(\nu)}{f_{r}^{2}}=\frac{\int_{V_{h}} S_{\epsilon}(\vec{r}, \nu, T)|\vec{E}|^{4} d \vec{r}}{4\left(\int_{V} \epsilon|\vec{E}|^{2} d \vec{r}\right)^{2}} .
$$

If $S_{\epsilon}$ is independent of the field strength $|\vec{E}|$, Eq. (5) predicts that the resonator noise is independent of microwave power, contrary to our observations ${ }^{3,7,8}$ which are made at the relatively high power levels of interest for detector applications. As we have argued previously, ${ }^{7}$ TLS saturation effects are very likely responsible for the observed power dependence of the noise. The saturation of TLS dissipation is a wellknown effect; ${ }^{10-12}$ we therefore make the ansatz that the noise depends on field strength in a similar manner:

$$
S_{\epsilon}(\vec{r}, \nu, \omega, T)=\kappa(\nu, \omega, T) / \sqrt{|\vec{E}(\vec{r})|^{2}+E_{n, c}^{2}(\omega, T)},
$$

where $E_{n, c}(\omega, T)$ is a critical electric field, likely related to the critical field for the saturation of the TLS dissipation, and the noise spectral density coefficient $\kappa(\nu, \omega, T)$ is allowed to vary with (microwave) frequency $\omega$ and temperature. ${ }^{3} \mathrm{Be}-$ cause we are assuming a uniform distribution of TLS in the volume $V_{h}$, we do not expect $S_{\epsilon}$ to have an additional explicit dependence on position $\vec{r}$. At high power for which $E$ $\gg E_{n, c}$ in the region contributing significantly to the resonator noise, Eq. (5) becomes

$$
\frac{S_{\delta f}(\nu)}{f_{r}^{2}}=\kappa(\nu, \omega, T) \frac{\int_{V_{h}}|\vec{E}|^{3} d^{3} r}{4\left(\int_{V} \epsilon|\vec{E}|^{2} d^{3} r\right)^{2}},
$$

which exhibits the desired $P_{\text {int }}^{-1 / 2} \propto 1 /|\vec{E}|$ scaling with power.

Equation (7) implies that the noise contributions are weighted by $|\vec{E}|^{3}$, so TLS fluctuators located near the coupler end of a quarter-wave resonator should give significantly larger noise contributions than those located near the shorted end. Therefore, for the resonators that are wider than the coupler $\left(s_{r}>3 \mu \mathrm{m}\right)$, the measured values of $S_{\delta f} / f_{r}^{2}$ need to be corrected for the coupler's noise contribution. A similar procedure was applied in Paper A to correct the frequency shift data. In the limit $l_{c} \ll l_{r}$, the correction is given by $S^{*}$ jsp 


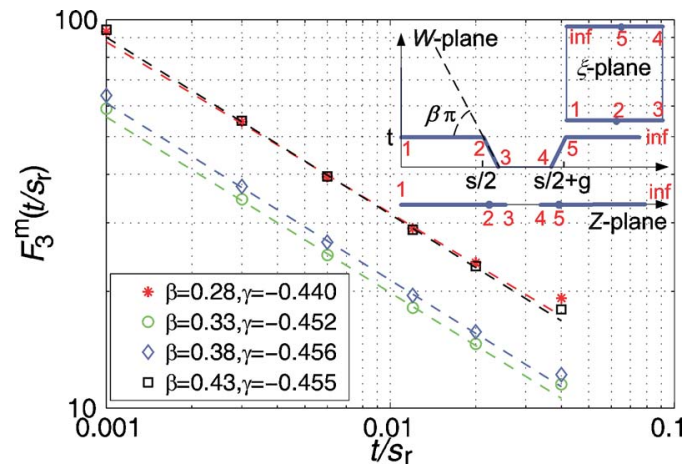

FIG. 3. (Color online) The calculated dimensionless noise scaling function $F_{3}^{m}\left(t / s_{r}\right)$ is plotted as a function of the ratio between the CPW half film thickness $t$ and the center strip width $s_{r}$. The inset shows the conformal mapping used to derive the electric field. The contour integral for $F_{3}^{m}\left(t / s_{r}\right)$ is evaluated on the surface of the metal, as outlined by the solid lines in the $W$-plane. Results are shown for four different values of the parameter $\beta$ $=0.28,0.33,0.38,0.43$ that controls the edge shape (see inset). The dashed lines indicate power law $\left(t / s_{r}\right)^{\gamma}$ fits to $F_{3}^{m}\left(t / s_{r}\right)$.

$=\left(S_{\delta f}-\eta S_{\delta f, 3 \mu \mathrm{m}}\right) /(1-\eta)$, where $\eta=3 \pi l_{c} / 4\left(l_{c}+l_{r}\right)$. The corrected values are plotted in Fig. 2 and are found to have a simple power-law scaling $1 / s_{r}^{1.58}$. We find a similar noise scaling, $1 / s_{r}^{\alpha}$ with $\alpha$ between 1.49 and 1.6, for noise frequencies $400 \mathrm{~Hz}<\nu<3 \mathrm{kHz}$.

While the fact that an $|E|^{3}$-weighted coupler noise correction leads to a simple power law noise scaling is already quite encouraging, we will now go further and show that the observed $s_{r}^{-1.58}$ power-law slope can be reproduced by our model. Measurements of the anomalous low-temperature frequency shift described in Paper A have already pointed to a surface distribution of TLS. If these TLS are also responsible for the frequency noise, according to Eq. (7) we would expect the noise to have the same geometrical scaling as the contour integral $I_{3}=\int|\vec{E}|^{3} d s$ evaluated either on the metal surface $\left(I_{3}^{m}\right)$ or the exposed substrate surface $\left(I_{3}^{g}\right)$. For zerothickness CPW, although the integral is divergent, the expected scaling can be shown to be $I_{3} \propto 1 / s_{r}^{2}$. For CPW with finite thickness, we can evaluate $I_{3}$ numerically using the electric field derived from a numerical conformal mapping solution. The two-step mapping procedure used here is modified from that given by Collin ${ }^{3}$ and is illustrated in the inset of Fig. 3. We first map a quadrant of finite-thickness CPW with half thickness $t$ (in the $W$ plane) to a zero-thickness CPW (in the $Z$ plane) and then to a parallel-plate capacitor (in the $\xi$ plane). To avoid nonintegrable singularities, we must constrain all internal angles on the conductor edges to be less than $\pi / 2$, which leads to the condition $0.25<\beta$ $<0.5$, where $\beta \pi$ is the angle defined in Fig. 3 .

Instead of evaluating $I_{3}$ directly, we define a normalized dimensionless integral $F_{3}\left(t, s_{r}\right)=\int\left|\vec{E} / E^{*}\right|^{3} d s^{*}$, where $s^{*}$ $=s / s_{r}$ is a normalized integration coordinate and $E^{*}=V / s_{r}$ is a characteristic field strength for a CPW with voltage $V$. Now $F_{3}$ depends only on the ratio $t / s_{r}$ and is related to the original contour integral by $I_{3}\left(s_{r}, t, V\right)=\left(V^{3} / s_{r}^{2}\right) F_{3}\left(t / s_{r}\right)$. The results $F_{3}^{m}\left(t / s_{r}\right)$ calculated for the metal surface are plotted in Fig. 3, and show a power-law scaling $F_{3}^{m} \sim\left(t / s_{r}\right)^{\gamma}$ with $\gamma \approx-0.45$ for $0.003<t / s_{r}<0.02$, the relevant range for our experiment. We also find that for a wide range of $\beta, 0.27<\beta$ $<0.43$, although the absolute values of $F_{3}^{m}\left(t / s_{r}\right)$ vary signifi- cantly, the scaling index $\gamma$ remains almost constant, -0.456 $<\gamma<-0.440$. Therefore, $\gamma$ appears to depend little on the edge shape.

From Eq. (7), the noise scaling is predicted to be $I_{3}^{m}\left(t, s_{r}, V\right) \propto s_{r}^{-2-\gamma} \sim s_{r}^{-1.55}$ (at fixed $V$ ), which agrees surprisingly well with the measured $s_{r}^{-1.58}$ scaling. We also investigated the case for TLS located on the exposed substrate surface, and found that $F_{3}^{g}$ has almost identical scaling $(\gamma$ $\approx-0.45)$ as $F_{3}^{m}$. While we still cannot say whether the TLS are on the surface of the metal or the exposed substrate, we can safely rule out a volume distribution of TLS fluctuators in the bulk substrate; this assumption yields a noise scaling of $\sim s_{r}^{-1.03}$, significantly different than measured.

In summary, the scaling of the frequency noise with resonator power and CPW geometry can be satisfactorily explained by a semiempirical model assuming a surface distribution of independent TLS fluctuators. These results allow the resonator geometry to be optimized. For example, one can design a quarter-wave CPW kinetic inductance detector $^{1,2}$ which is wider on the coupler end to benefit from the noise reduction, but narrower at the low- $|\vec{E}|$ shorted end to maintain a high kinetic inductance fraction and responsivity. If the spatial distribution of the TLS and the $\vec{E}$ field are both known, values of $\kappa$ and $E_{n, c}$ can be determined, allowing noise predictions to be made using Eq. (7). Unfortunately, we do not know the exact $\vec{E}$ field distribution for our CPW resonators because of the sensitivity to the edge shape, nor do we know the thickness of the TLS surface layer. Future experiments with simplified geometries and at lower powers should allow our ansatz [Eq. (6)] to be tested, and may yield a quantitative determination of $\kappa(\nu, \omega, T)$.

We thank Clare Yu and Sunil Golwala for useful discussions. The device was fabricated in the University of California, Berkeley, Microfabrication Laboratory. This work was supported in part by the NASA, NSF, JPL, and the Gordon and Betty Moore Foundation.

${ }^{1}$ B. A. Mazin, P. K. Day, J. Zmuidzinas, and H. G. LeDuc, AIP Conf. Proc. 605, 309 (2002).

${ }^{2}$ P. K. Day, H. G. LeDuc, B. A. Mazin, A. Vayonakis, and J. Zmuidzinas, Nature (London) 425, 817 (2003).

${ }^{3}$ S. Kumar, J. Gao, J. Zmuidzinas, B. A. Mazin, H. G. Leduc, and P. K. Day, Appl. Phys. Lett. 92, 123503 (2008).

${ }^{4}$ J. Gao, M. Daal, P. K. Day, B. A. Mazin, H. G. LeDuc, A. Vayonakis, S. Kumar, B. Sadoulet, and J. Zmuidzinas, Appl. Phys. Lett. 92, 152505 (2008).

${ }^{5}$ J. A. B. Mates, G. C. Hilton, K. D. Irwin, L. R. Vale, and K. W. Lehnert, Appl. Phys. Lett. 92, 023514 (2008).

${ }^{6}$ J. Baselmans, S. J. C. Yates, R. Barends, Y. J. Y. Lankwarden, J. R. Gao, H. Hoevers, and T. M. Klapwijk, J. Low Temp. Phys. 151, 524 (2008).

${ }^{7}$ J. Gao, J. Zmuidzinas, B. A. Mazin, P. K. Day, and H. G. LeDuc, Appl. Phys. Lett. 90, 817 (2007).

${ }^{8}$ J. Gao, B. Mazin, M. Daal, P. Day, H. LeDuc, and J. Zmuidzinas Proc. SPIE 6275, 627509 (2006).

${ }^{9}$ W. A. Phillips, J. Low Temp. Phys. 7, 351 (1972).

${ }^{10}$ S. Hunklinger and W. Arnold, Physical Acoustics (Academic, New York, 1976), Vol. 12, Chap. 3, p. 155.

${ }^{11}$ J. M. Martinis, K. B. Cooper, R. McDermott, M. Steffen, M. Ansmann, K. D. Osborn, K. Cicak, S. Oh, D. P. Pappas, R. W. Simmonds, and C. C. Yu, Phys. Rev. Lett. 95, 210503 (2005).

${ }^{12}$ W. A. Phillips, Rep. Prog. Phys. 50, 1657 (1987).

${ }^{13}$ R. E. Collin, Foundations for Microwave Engineering, 2nd ed. (IEEE, New York, 2000). 